\title{
Erosion spécifique et caractérisation de la résistance au cisaillement des sédiments du barrage de Fergoug
}

\author{
Belkacem MEKERTA ${ }^{1}$, Abdélaziz SEMCHA ${ }^{1}$, Fadéla RAHMANI ${ }^{1}$, \\ Jean Pierre TROALEN ${ }^{2}$ \\ ${ }^{1}$ Ecole Normale Supérieure d'Enseignement Technique, ENSET Oran, \\ Laboratoire "LABMAT", Département Génie Civil \\ B.P. 1523 Oran El M'Naouer 31000, Algérie \\ mekerta.b@enset-oran.dz
}

${ }^{2}$ Université de Reims Champagne Ardenne, UFR Sciences Exactes et Naturelles, Moulin de la Housse, BP 1039, 51687, Reims Cedex, France

jp.troalen@univ-reims.fr

\begin{abstract}
Résumé :
L'étude présentée porte sur la caractérisation de la résistance au cisaillement des sédiments d'envasement de la retenue du barrage de Fergoug situé au nord ouest de l'Algérie. En premier, la quantification des apports solides sous forme d'érosion spécifique est présentée par une analyse probabiliste. Ensuite, à partir de sondages réalisés le long des principaux affluents et au pied du barrage, des carottes ont été prélevées. Dans le but de localiser les zones à cibler pour le dragage, la connaissance de la variation de la résistance au cisaillement est nécessaire. A partir des valeurs obtenues sur des échantillons sélectionnés, nous présentons dans une seconde étape la cohésion non drainée au niveau de chaque sondage et pour des tranches de profondeurs choisies.
\end{abstract}

\begin{abstract}
:
The presented study relates to the characterization of the shear strength of the silting sediments of the Fergoug's dam located at the western north of Algeria. In first, the estimate of solid matters as specific erosion is presented with a probability method. After that, from surveys carried out along the main affluents and at the foot of the dam, samples were taken. In order to locate the significant zones for dredging, knowing the distribution of the shear strength is necessary. Starting from some values obtained on samples selected, we present in second time the undrained cohesion for every survey and for some selected depth slices.
\end{abstract}

Mots-clés :

Barrage - Erosion spécifique - Cohésion non drainée - Dragage

DOI: 10.5150/jngcgc.2008.013-M (disponible en ligne - http://www.paralia.fr - available online) 


\section{Introduction}

L'alluvionnement des retenues de barrages est un risque sérieux pour la durée de vie de celles-ci. Ce problème est plus critique dans les pays à climat aride ou semi-aride. L'Algérie perd annuellement un volume de stockage d'eau de 32 millions de $\mathrm{m}^{3}$ sur un volume de 5,2 milliards de $\mathrm{m}^{3}$ des 114 barrages en exploitation. Les terrains vulnérables de formations géologiques marneuses sont soumis à une érosion accélérée. Notre étude porte sur le site du barrage de Fergoug qui est une illustration concrète des aires privilégiées de dépôt des matières solides. Contraints de préserver les volumes utiles des barrages, les gestionnaires ont eu recours à des opérations de dragage des sédiments. Le choix des méthodes d'enlèvement à adopter nécessite la connaissance des caractéristiques géotechniques des matières solides accumulées au fond des retenues. Des sondages profonds allant de 6 à $28 \mathrm{~m}$ ont été exécutés par l'Entreprise Nationale de Réalisation des Barrages, sur les principaux affluents et jusqu'au pied du barrage sur une distance de $2 \mathrm{~km}$ environ.

Nous allons présenter les résultats de l'état de l'alluvionnement du sous bassin versant de l'oued Fergoug et l'identification géotechnique de la résistance au cisaillement des sédiments prélevés. Dans cette contribution, en plus des informations complémentaires sur le volume des dépôts devant être dragués, nous exposons la variation de ce paramètre géotechnique qui permet la connaissance $\mathrm{du}$ comportement des sédiments en différents lieux par rapport aux organes du barrage, dans le but du choix des moyens de dragage adaptés à l'état des sédiments en place.

\section{Caractéristiques de l'ouvrage et de la retenue}

\subsection{Situation géographique}

Le barrage de Fergoug montré sur la figure 1, est situé au nord-ouest de l'Algérie (sur l'Oued El Hammam), au pied des contreforts de l'Atlas à environ $20 \mathrm{~km}$ au sud de la ville de Mohammadia (Wilaya de Mascara) à $80 \mathrm{~km}$ d'Oran, et en aval du confluent de l'Oued El Hammam avec le barrage de Bouhanifia. Ce barrage est construit en dessous de la réunion de l'Oued El Hammam, de l'Oued Gharzout et de l'Oued Fergoug (voir figure 2). Le bassin versant de la retenue du Fergoug représente une superficie totale estimée à $8340 \mathrm{~km}^{2}$. L'altitude moyenne est de $790 \mathrm{~m}$ avec comme altitude maximale $1454 \mathrm{~m}$.

\subsection{Problème particulier du barrage de Fergoug}

Dans son état actuel, le barrage de Fergoug est un ouvrage en terre de capacité initiale en 1970, de 17 millions de $\mathrm{m}^{3}$. Au bout de sept années (en 1977), sa capacité a chuté à 9,67 millions de $\mathrm{m}^{3}$, soit un taux annuel d'envasement dépassant un million de $\mathrm{m}^{3}$.

Le barrage du Fergoug assure la régularisation des Oueds Fergoug, El Hammam et Gharzout. Il assurait l'alimentation en eau potable des villes d'Oran, d'Arzew et Mohammadia, ainsi que l'alimentation en eau industrielle de la ville d'Arzew et l'irrigation de la plaine de Habra (20 000 ha). Actuellement, le barrage n'alimente 
que la commune de Béthioua du fait que son volume utile de stockage est devenu très réduit par l'accumulation des sédiments.

En raison de la perte à grande vitesse de son volume utile (MTPC, 1970), le barrage a subi des opérations de dragage dont la plus importante remonte à 1986 où 10 millions de $\mathrm{m}^{3}$ de vase ont été extraits. Cette opération a été à l'origine d'importants désordres sur l'environnement. Nous indiquons sur le plan d'ensemble du barrage de la figure 2 la localisation des sondages.
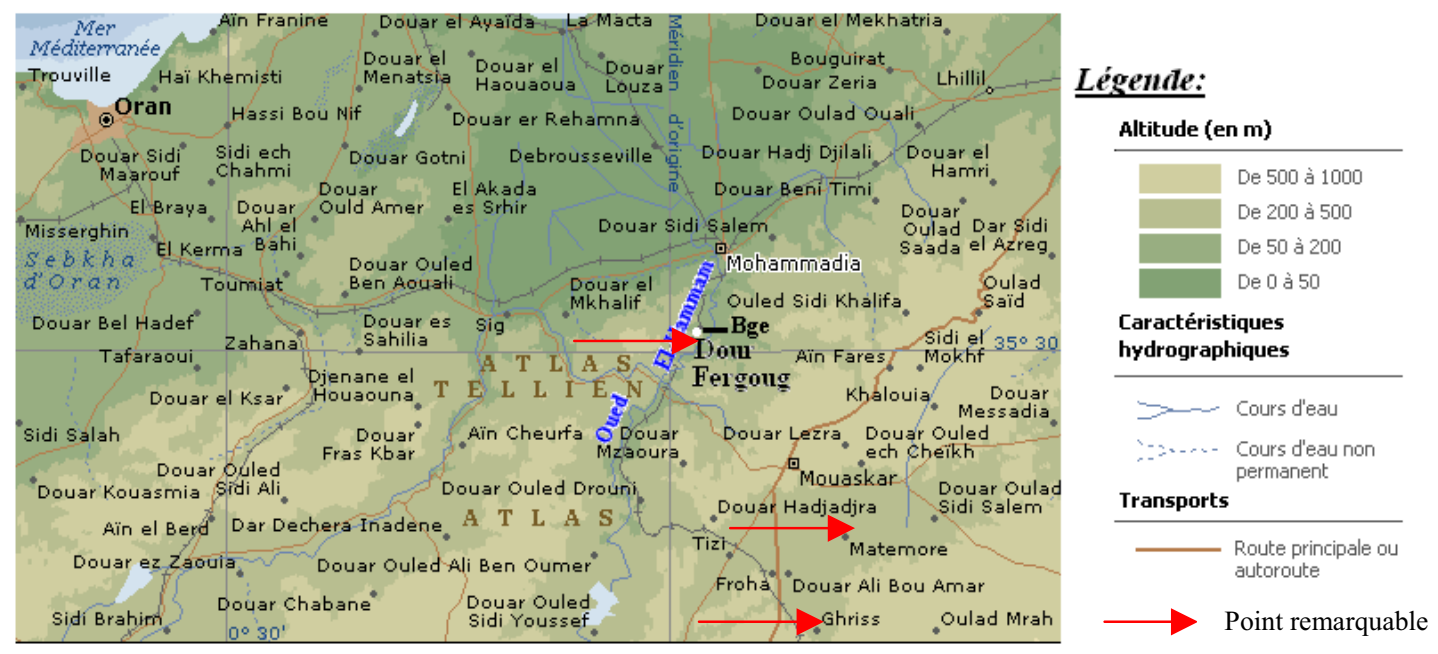

Figure 1. Situation géographique du barrage de Fergoug

\subsection{Localisation du sous bassin versant de l'oued Fergoug}

Le sous bassin versant de l'oued Fergoug, couvre une superficie de $122 \mathrm{~km}^{2}$ (voir figure 3), il fait partie du grand bassin versant de l'Oranie - Chott Chergui. La complexité de la région concernée est caractérisée par la prédominance des charriages qui marquent la région par un relief disséqué et vigoureux dans le détail. Au sein de cette structure tourmentée, des familles lithologiques peu résistantes ou franchement tendres prédominent. La quasi totalité des terrains est marquée par des séries marneuses datant du Crétacé ou du Néogène.

La lithologie du sous bassin versant de l'oued Fergoug fait apparaître une grande diversité des formations superficielles avec prédominance des sols argileux issus des formations marneuses (BOUCHETATA. A \& BOUCHETATA.T, 2006). 


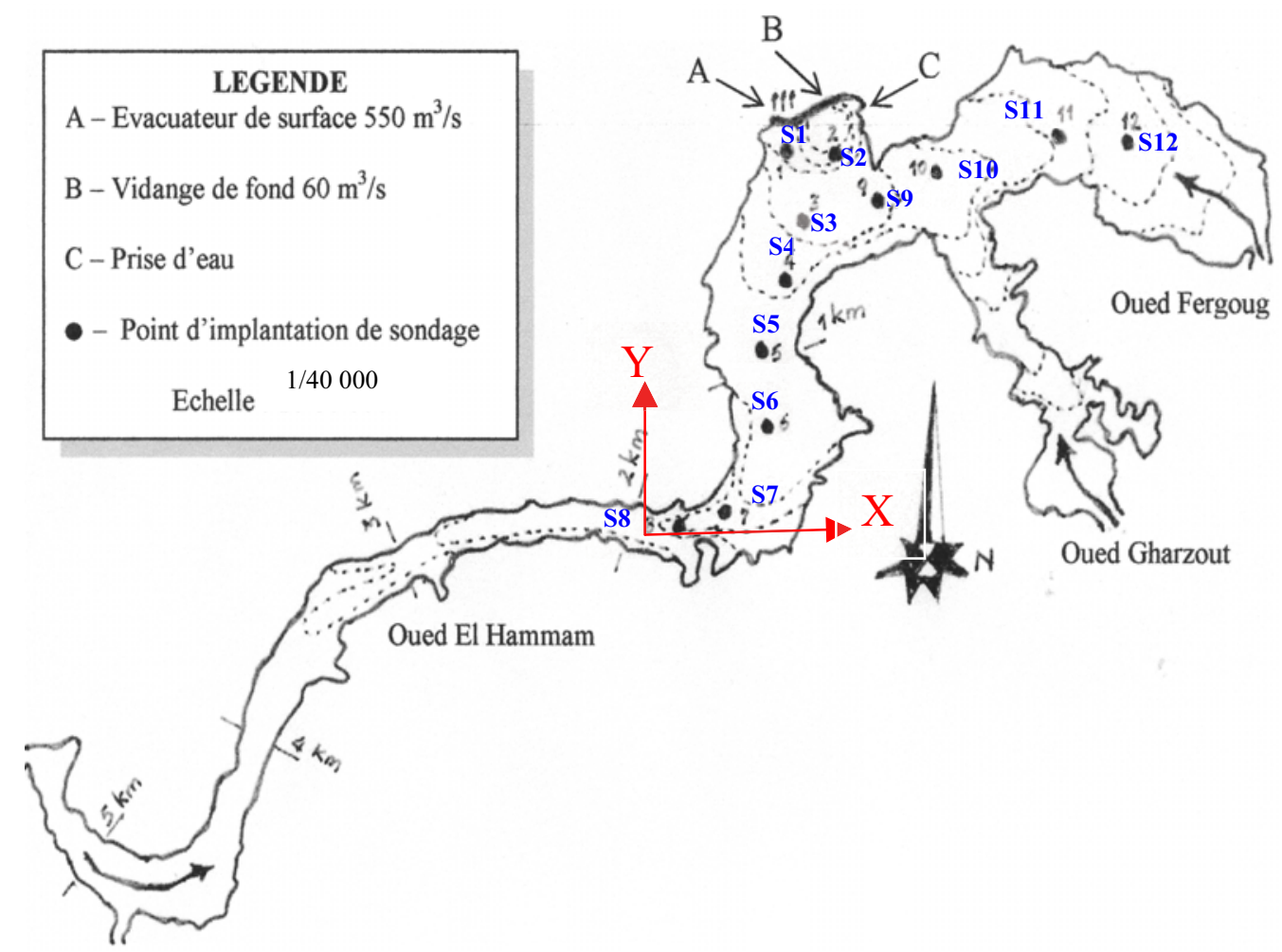

Figure 2. Plan d'ensemble du barrage de Fergoug - Implantation des sondages

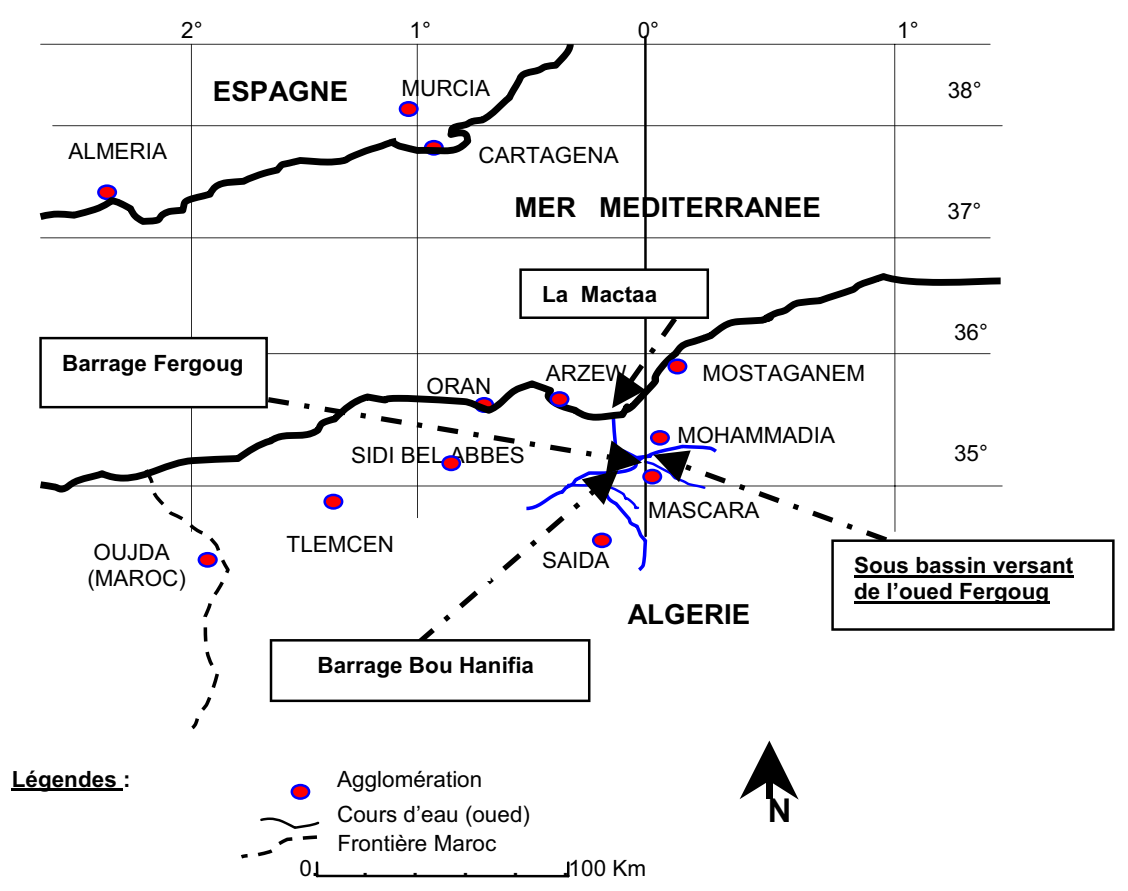

Figure 3. Carte de localisation du sous bassin versant de l'oued Fergoug 


\subsection{Estimation de l'érosion spécifique dans le sous bassin versant de l'oued Fergoug}

La zone concernée du sous bassin versant noté SBV, de l'oued Fergoug présente un relief très escarpé avec de fortes pentes supérieures à 0,5 sur une surface totale de $20,3 \%$. Les pentes supérieures à 0,25 concernent $35,3 \%$ de la surface totale et le reste de la surface $(61,5 \%)$ présente une pente supérieure à 0,125 . Les données des précipitations annuelles fournies par l'Institut Hydrométéorologique de Formation et de Recherche d'Oran, pour une période de 34 ans (1970 à 2003), pour les deux stations de Ghriss et de Matemore proches du SBV, ont permis d'estimer l'érosion spécifique annuelle. FOURNIER (in OLIVRY, 1991) a proposé une formule (1) en prenant en compte les précipitations et la morphologie $\mathrm{du}$ bassin versant. La nature des précipitations et les caractéristiques morphologiques du SBV étudié permettent l'application de cette formule (SEMCHA, 2006).

$\mathrm{E}_{\mathrm{S}}=(1 / 36) \cdot\left(\mathrm{Ps}^{2} / \mathrm{Pa}\right)^{2.65} \cdot\left(\mathrm{H}^{2} / \mathrm{S}\right)^{0.46}$

où :

$\mathrm{E}=$ apport solide annuel spécifique $\left(\mathrm{T} / \mathrm{km}^{2} / \mathrm{an}\right)$,

Ps = pluie mensuelle moyenne du mois le plus pluvieux ( $\mathrm{mm})$,

$\mathrm{Pa}=$ pluie annuelle $(\mathrm{mm})$,

$\mathrm{H}=$ pente moyenne,

$\mathrm{S}=$ superficie du SBV de l'oued Fergoug $\left(\mathrm{S}=122 \mathrm{~km}^{2}\right)$.

Pour les deux stations Ghriss et Matemore, nous avons calculé l'érosion spécifique par an en fonction de chaque pente du SBV de l'oued Fergoug $(0,5$; $0,25$ et 0,125$)$. Les figures 4 et 5 illustrent l'influence de la pluie sur l'érosion spécifique.

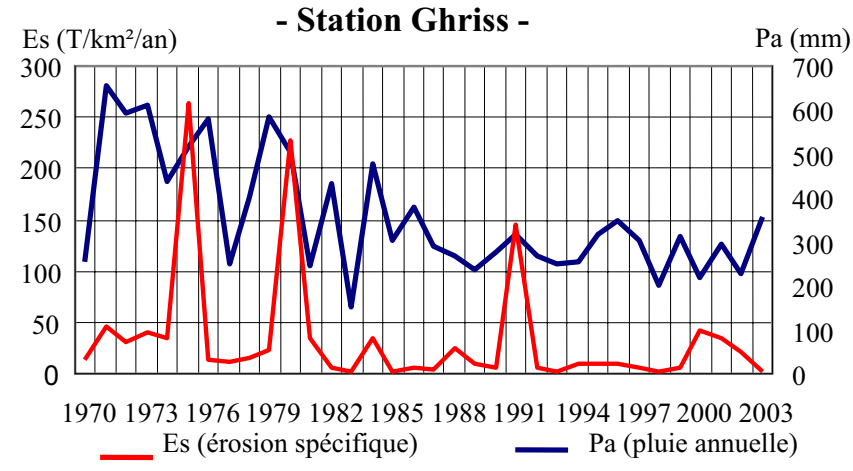

Figure 4. Evolution du taux d'érosion spécifique Es et de la pluie annuelle Pa en fonction du temps du SBV de l'oued Fergoug, (Station Ghriss) 


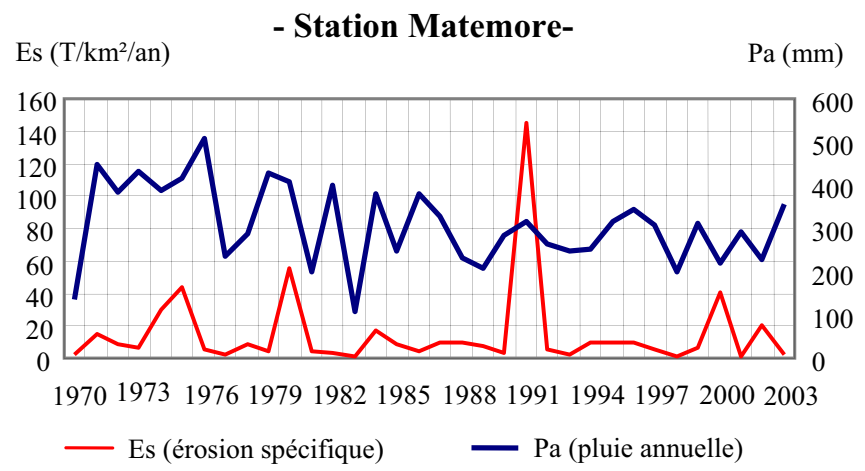

Figure 5. Evolution du taux d'érosion spécifique Es et de la pluie annuelle Pa en fonction du temps du SBV de l'oued Fergoug, (Station Matemore)

Nous remarquons sur les figues 4 et 5 une irrégularité du taux d'érosion spécifique et de la pluie annuelle en fonction des années d'observation au niveau des deux stations et une difficulté à corréler ces deux paramètres. Ceci nous conduit à rechercher d'autres facteurs, notamment la nature de la pluie (temps de concentration et intensité), les effets saisonniers ainsi que l'irrégularité annuelle des précipitations exprimée par la succession des années humides et sèches.

Les figures 6 et 7 montrent la distribution de la probabilité de l'érosion pour différents bassins versants, DEMMAK (in MEKERTA, 1995), dans lesquelles nous avons inséré celles de l'érosion spécifique de notre SBV calculée sur une période de 34 ans (1970-2003) au niveau des deux stations Ghriss et Matemore. Dans les deux cas nous obtenus des droites de pentes voisines de celle donnée par DEMMAK pour les rivières de l'Afrique du nord.

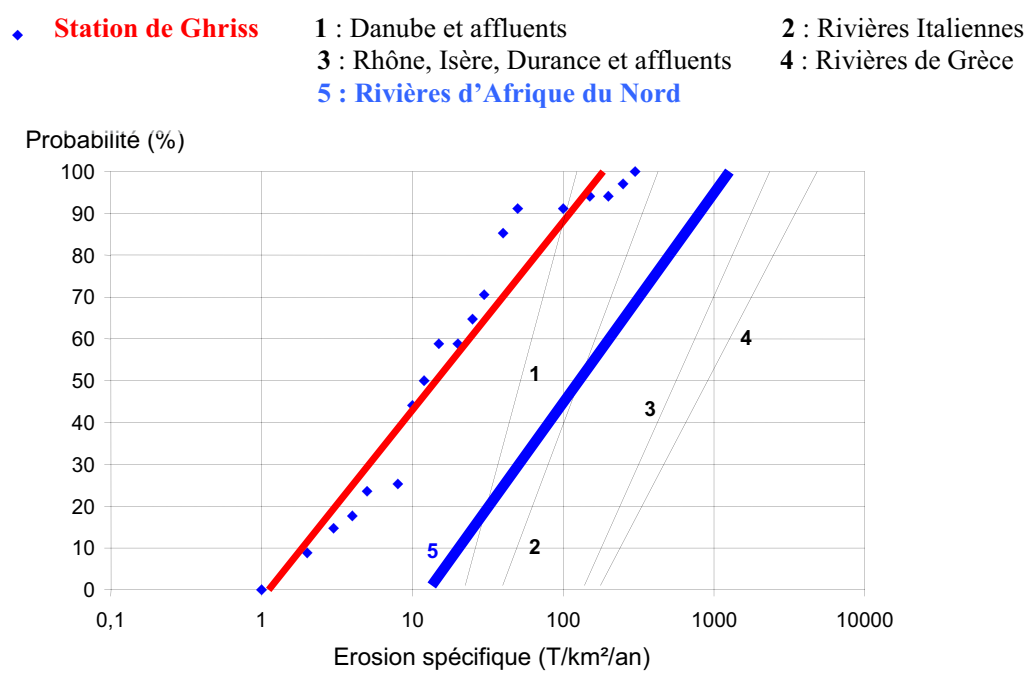

Figure 6. Distribution de l'érosion pour différents bassins versants 


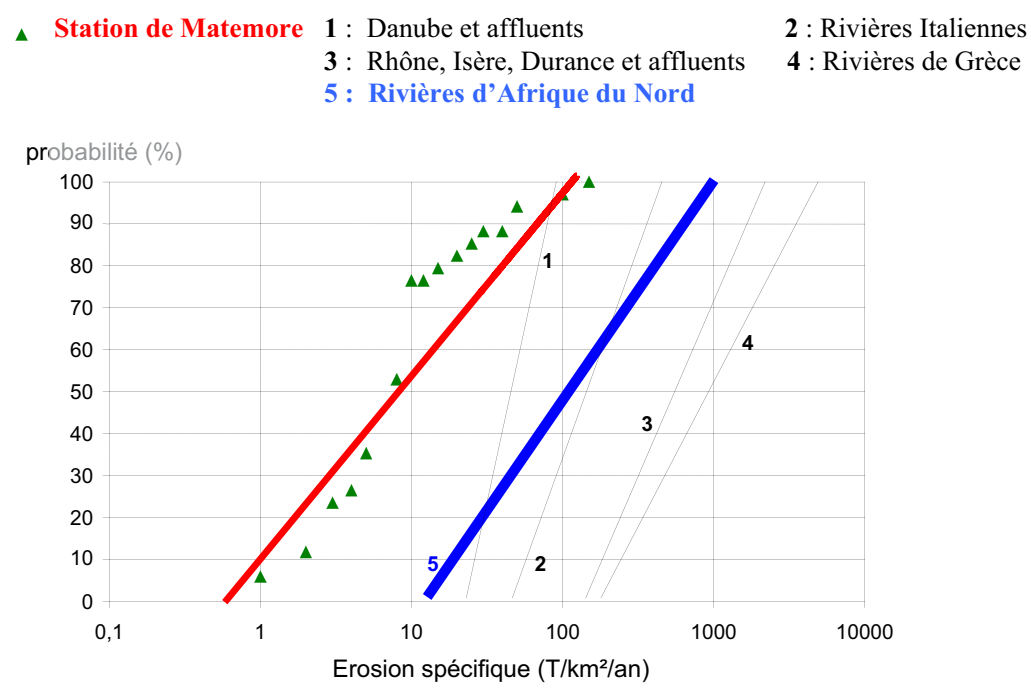

Figure 7. Distribution de l'érosion pour différents bassins versants

\section{Sondages et essais réalisés}

Pour une bonne connaissance de la répartition spatiale et l'analyse statistique des propriétés géomécaniques des sédiments, les 12 sondages et les organes du barrage ont été positionnés par rapport aux coordonnées $\mathrm{X}$ et $\mathrm{Y}$ d'un point fixe choisi, situé à $2 \mathrm{~km}$ sur la rive droite (voir figure 2 et tableau 1).

Tableau 1. Coordonnées des sondages et organes du barrage

\begin{tabular}{|c|c|c|c|c|c|}
\hline $\begin{array}{c}\boldsymbol{N}^{\circ} \\
\text { Sondage }\end{array}$ & $\boldsymbol{A}$ & $\boldsymbol{B}$ & $\boldsymbol{C}$ & $\boldsymbol{S 1}$ & $\boldsymbol{S 2}$ \\
\hline$X(\mathrm{~m})$ & 10651,28 & 10894,74 & 11030,43 & 10706,99 & 10921,96 \\
\hline$Y(\mathrm{~m})$ & 31609,04 & 31667,21 & 31660,75 & 31483,45 & 31470,81 \\
\hline $\begin{array}{c}\boldsymbol{N}^{\circ} \\
\text { Sondage }\end{array}$ & $\boldsymbol{S 3}$ & $\boldsymbol{S 4}$ & $\boldsymbol{S 5}$ & $\boldsymbol{S 6}$ & $\boldsymbol{S 7}$ \\
\hline$X(\mathrm{~m})$ & 10783,69 & 10700,76 & 10599,31 & 10625,39 & 10442,26 \\
\hline$Y(\mathrm{~m})$ & 31215,54 & 10993,52 & 30731,98 & 30443,36 & 30119,02 \\
\hline $\begin{array}{c}\boldsymbol{N}^{\circ} \\
\text { Sondage }\end{array}$ & $\mathbf{S 8}$ & $\boldsymbol{S 9}$ & $\boldsymbol{S 1 0}$ & $\boldsymbol{S 1 1}$ & $\boldsymbol{S 1 2}$ \\
\hline$X(\mathrm{~m})$ & 10246,55 & 11095,20 & 11345,05 & 11871,20 & 12167,29 \\
\hline$Y(\mathrm{~m})$ & 30068,86 & 31291,12 & 31399,11 & 31523,93 & 31498,25 \\
\hline
\end{tabular}

$\boldsymbol{A}, \boldsymbol{B}, \boldsymbol{C}:$ organes du barrage. $\quad \boldsymbol{S} 1$ à $\boldsymbol{S 1 2}:$ sondages

\section{Variation de la cohésion non drainée}

Parmi les paramètres étudiés nous présentons dans cette contribution la variation de la cohésion non drainée $(\mathrm{Cu})$. Les valeurs de $\mathrm{Cu}$ ont été obtenues à partir d'essais de cisaillement direct à la boîte de Casagrande non consolidé non drainé. Les échantillons sont sélectionnés dans les carottes provenant des sondages en fonction de la profondeur

L'étude de la variation de la cohésion non drainée des sédiments ou toute autre propriété géomécanique, apparaît comme préalable à toute tentative de modélisation visant une meilleure compréhension des phénomènes d'envasement. 
La variation de ce paramètre est fonction de la position du point considéré en profondeur et en plan. Pour cela, nous avons considéré des tranches de profondeurs allant de : 0 à $5 \mathrm{~m}, 5$ à $15 \mathrm{~m}$ et plus de $15 \mathrm{~m}$. L'épaisseur de chaque tranche a été fonction du nombre des essais réalisés (la tranche de profondeur peut être modifiée).

Les figures 8, 9 et 10 représentent les variations de la cohésion non drainée pour chacune des tranches considérées.

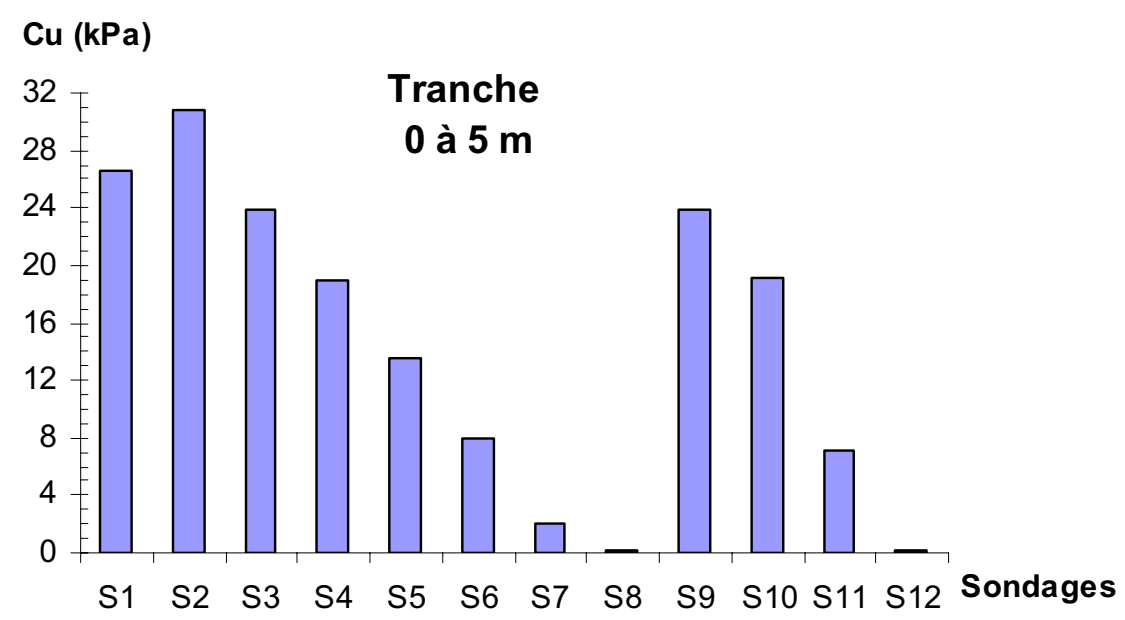

Figure 8. Représentation graphique de la cohésion non drainée (tranche 0 à $5 \mathrm{~m}$ )

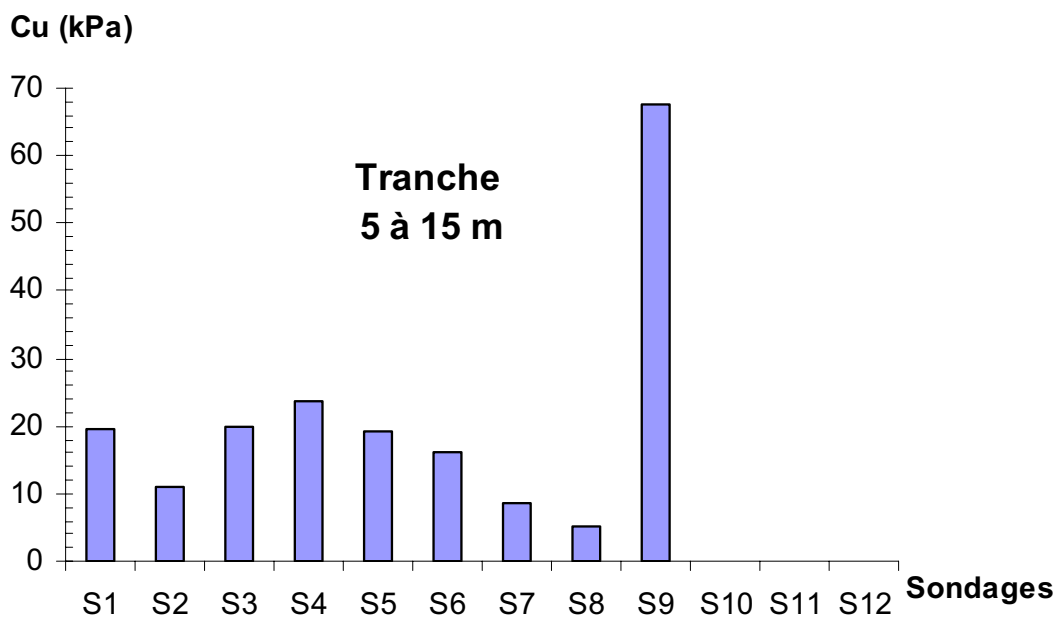

Figure 9. Représentation graphique de la cohésion non drainée (tranche 5 à 15m) 


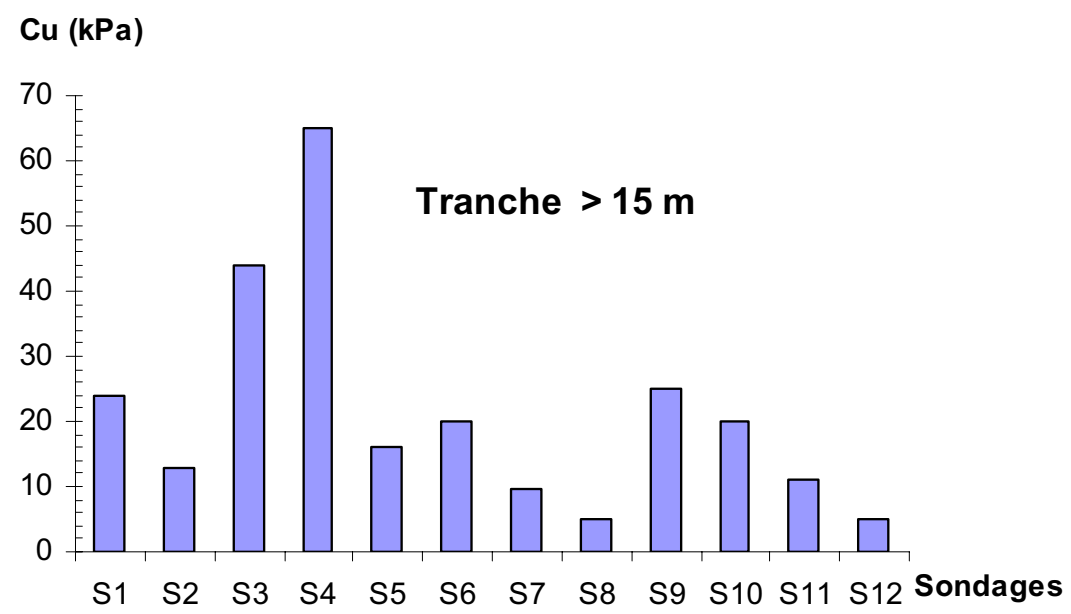

Figure 10. Répartition spatiale de la cohésion non drainée (tranche $>15 \mathrm{~m}$ )

La résistance au cisaillement permet de caractériser l'état "mécanique » du matériau vis-à-vis d'une sollicitation instantanée.

Si nous considérons globalement les résultats de la cohésion non drainée en fonction des tranches de profondeurs telles que représentées sur les figures 8,9 et 10 , nous devrions limiter des fourchettes de valeurs de l'ordre de $25 \mathrm{kPa}$ pour les profondeurs 0 à $5 \mathrm{~m}$ concernant les sondages voisins de la digue. De même pour la tranche 5 à $15 \mathrm{~m}$ les valeurs sont de l'ordre de $20 \mathrm{kPa}$. Par contre en s'éloignant de la digue nous relevons des valeurs de $\mathrm{Cu}$ proches de $65 \mathrm{kPa}$ pour la tranche de profondeurs supérieure à $15 \mathrm{~m}$.

Ces variations constatées montrent l'existence de relations entre l'état de consolidation des sédiments en place et des variations lithologiques.

\section{Conclusion}

La quantification des apports solides par les études probabilistes de l'érosion spécifique effectuée sur le sous bassin versant de l'oued Fergoug, a confirmé la grande irrégularité de l'érosion. Il s'agit en effet d'une région à climat semi-aride dans laquelle l'érosion est particulièrement active et irrégulière suivant les variations pluviométriques annuelles et saisonnières. D'autres facteurs tels que la nature de la pluie (le temps de concentration, l'intensité) et la nature du sol doivent être pris en considération.

D'autre part, la représentation graphique de la cohésion non drainée $\mathrm{Cu}$ montre une certaine variabilité mécanique pour les tranches de profondeurs choisies. En effet, nous notons clairement une tendance à la diminution de ce paramètre en s'éloignant de la digue du barrage et un regroupement des plus importantes valeurs au voisinage du barrage. Ces résultats peuvent être exploités pour la localisation des zones à draguer en priorité vis-à-vis des organes du barrage. 


\section{$6 \underline{\text { Références bibliographiques }}$}

1 BOUCHETATA A., BOUCHETATA T. (2006). Propositions d'aménagement du sous bassin versant de l'oued Fergoug (Algérie) fragilisé par des épisodes de sécheresse et soumis à l'érosion hydrique. Sécheresse 2006, 17 (3), pp 415-424.

2 MTPC. (1970). Direction de l'hydraulique du Ministère des Travaux Publics et de la Construction, Algérie. Note sur l'état en 1/1/1970 des études et réalisations pour la lutte contre l'envasement des barrages Algériens, Agence Nationale des Barrages, $30 \mathrm{p}$.

3 MEKERTA B., (1995). Etude des propriétés géomécaniques des sédiments d'envasement du barrage de Génissiat. Thèse, Institut National Polytechnique de Lorraine, $196 \mathrm{p}$.

4 OLIVRY J.C. (1991). Cours d'initiation à l'étude et la mesure de l'érosion et des transports solides. Cah. ORSTOM. Sér. Pédol., vol. XXV, 110 p.

5 SEMCHA A., (2006). Valorisation des sédiments issus des dragages. Applications dans le BTP (cas de barrage algérien). Thèse de l'Université de Reims Champagne Ardenne, $171 \mathrm{p}$. 